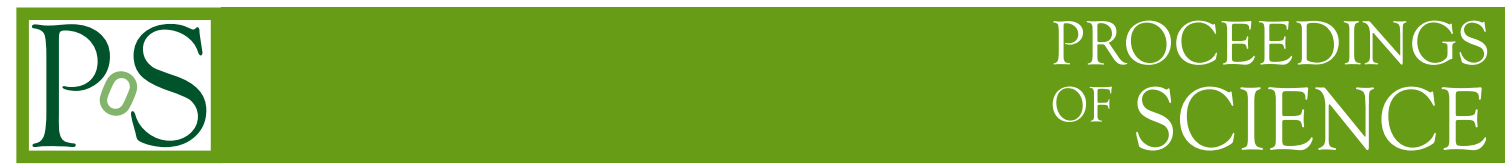

\title{
Jets and Jet Substructure at an EIC
}

\section{B. S. Page*}

\section{Brookhaven National Laboratory}

E-mail: bpageabn 1 .gov

The goal of the planned high-energy high-luminosity polarized electron-ion collider (EIC) is a detailed understanding of the QCD dynamics that underlies the nucleons and nuclei. With advances in experimental technique and theoretical understanding over the past several decades, jets have become precision tools in the exploration of QCD in collider environments. Therefore, precision jet measurements have the potential to be important components of the electron-hadron and electron-nucleus EIC physics programs. One property of jets that may prove especially useful is that their substructure, i.e. their internal energy distribution, can be rigorously defined and studied systematically. This contribution will discuss possible substructure observables at an EIC as well as outline their applications and experimental aspects of their measurement.

XXVII International Workshop on Deep-Inelastic Scattering and Related Subjects - DIS2019 8-12 April, 2019

Torino, Italy

\footnotetext{
* Speaker.
} 


\section{Introduction}

Jet substructure observables, which describe the spacial distribution of energy within a jet, have emerged as important tools at collider experiments in many different contexts. In $p p$ collisions at the LHC, substructure can be used to identify heavy boosted objects or discriminate between jets arising from quarks versus those from gluons (see [1, 2]). Substructure has also found an application in AA collisions at both RHIC and the LHC where their sensitivity to the dispersal of energy throughout the jet can be used to study the interaction between hard partons and the hot and dense nuclear medium formed in relativistic heavy ion collisions [3].

Evaluation of the potential utility of jets at the EIC is still in the early stages, and jet substructure has received even less attention. As in $A A$ collisions, it is hoped that substructure observables will be sensitive to the interactions between high momentum partons and the medium they traverse so that substructure can shed light on the properties of cold nuclear matter. Before their potential in $e A$ can be evaluated, substructure observables need to be first understood both experimentally and theoretically in $e p$ collisions to have a solid basis. This work is a first step in that direction.

The studies discussed below were performed using pseudo-data generated with the PYTHIA6.4 [4] Monte Carlo program using the CTEQ5m [5] PDF set. Both resolved and direct subprocesses were simulated and events were restricted to the photoproduction region $\left(10^{-5}<Q^{2}<\right.$ $1 \mathrm{GeV}^{2}$ ). The inelasticity was required to be between 0.2 and 0.8 . Jets were reconstructed in the laboratory frame from all stable final-state particles with transverse momenta greater than $250 \mathrm{MeV} / \mathrm{c}$ (or $500 \mathrm{MeV} / c$ ) and pseudorapidity between \pm 4.0 . Clustering was performed using the anti- $\mathrm{k}_{T}[6]$ algorithm as implemented in FastJet [7] using E_scheme recombination and two radius parameters $R: 0.4$ and 0.8 . The resulting jets were required to have transverse momenta greater than $5 \mathrm{GeV} / c$ and pseudorapidity between -2.0 and 4.0 , where positive pseudorapidity is defined to be in the hadron-going direction.

\section{Angularity Overview}

The substructure observable utilized for this study is the jet angularity [8], which is defined as:

$$
\tau_{a} \equiv \frac{1}{p_{\mathrm{T}}} \sum_{i \in J} p_{\mathrm{T}}^{i}\left(\Delta R_{i J}\right)^{2-a}
$$

where $p_{\mathrm{T}}$ and $p_{\mathrm{T}}^{i}$ are the transverse momenta of the jet and the $i^{\text {th }}$ particle within the jet, respectively, $\Delta R_{i J}$ is the distance in $\eta-\phi$ space between the $i^{\text {th }}$ jet particle and the jet thrust axis, and ' $a$ ' is the parameter that controls the sensitivity to collinear radiation. The sum runs over all particles within the jet. Different values of ' $a$ ' interpolate between traditional jet shape observables, with $a=0$ corresponding to jet mass and $a=1$ corresponding to broadening/girth.

Figure 1(a) presents the angularity spectrum for jets with $R=0.8$ and transverse momenta greater than $5 \mathrm{GeV} / c$ for resolved and direct events. Diffuse jets, with significant energy at large radii have larger angularity values while more collimated jets have smaller angularities as can be seen in Fig. 1(b), which shows the jet profile, or fraction of jet energy contained within a cone of radius $r<R$, for four different regions of $\log _{10}(\tau)$. As ' $a$ ' increases, the radial position of the particles is given less weight and the angularity distribution becomes more peaked and shifts to 


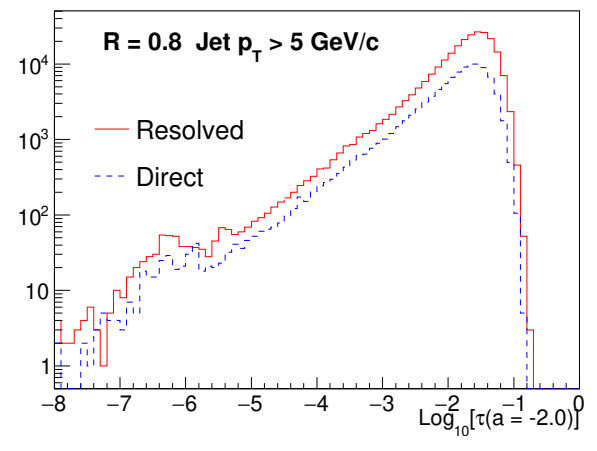

(a)

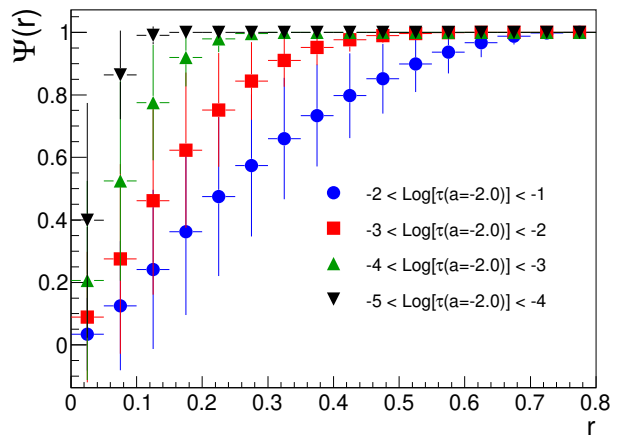

(b)

Figure 1: (a) The logarithm of angularity for jets with $R=0.8, p_{\mathrm{T}}>5 \mathrm{GeV} / c$, and $a=-2.0$ separated into resolved (red) and direct (blue) subprocesses. (b) Jet profiles (average energy contained within a radius $r<R$ ) for four ranges of the jet angularity spectrum in (a). The direct and resolved components are combined and the bars represent the RMS of the distributions.

larger values. Similarly, as the jet radius is decreased, $\Delta R_{i J}$ is restricted to smaller values and the angularity spectrum shifts to the left, although the shape remains largely unchanged.

\section{Detector Considerations}

The angularity spectrum in Fig. 1(a) was obtained at 'particle-level', that is the full information about each particle in the event was available to the jet finder. Of course, in an actual experiment, detector resolutions and inefficiencies will impact the determination of individual particle fourmomenta and thus affect the measured angularity spectra. A full study of detector effects will require dedicated GEANT modeling of realistic detector designs, however, a sense for the expected impacts can be obtained via alterations of the particle-level simulation used here. The first effect considered was the loss of low $p_{\mathrm{T}}$ particles due to, for example, excessive deflection in a solenoidal magnetic field or because of noise in calorimeter systems. The nominal particle $p_{\mathrm{T}}$ threshold for inclusion in a jet is $250 \mathrm{MeV} / c$ and was doubled to $500 \mathrm{MeV} / c$ for this study. The result on the angularity spectrum with $a=-2.0$ for jets with $R=0.8$ and $p_{\mathrm{T}}>5 \mathrm{GeV} / c$ can be seen in Fig. 2(a). It is seen that jets with the higher particle $p_{\mathrm{T}}$ threshold are somewhat more collimated as may be expected since lower $p_{\mathrm{T}}$ particles are fractionally more abundant at large radii.

A second issue studied is how the angularity spectrum is modified by the loss of neutrons and $K_{\mathrm{L}}^{0} \mathrm{~s}$, whose energy and position can only be determined via hadron calorimeters. The energy resolution of hadron calorimeters is generally poor, and this will be exacerbated by the low energy of the particles produced at an EIC. In addition, the extended nature of hadronic showers makes determining the precise direction of a neutral hadron with a calorimeter difficult. A realistic accounting of the impact these effects will have on the measured angularity will require a full detector simulation using a realistic hadron calorimeter model, which is beyond the scope of this work. However, a worst-case-scenario evaluation of the effect that poor hadron calorimeter resolution can have on an angularity measurement can be achieved by simply ignoring the neutral hadron component of the 


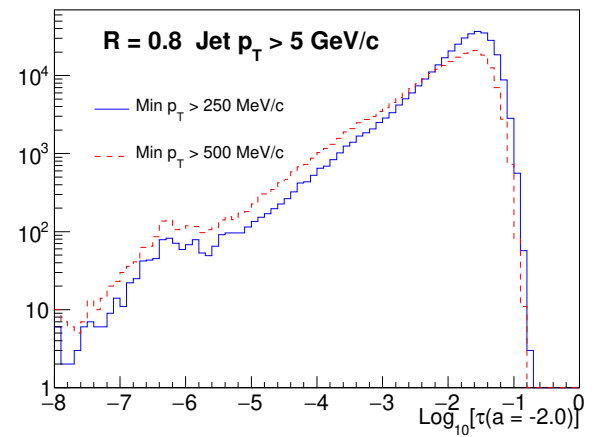

(a)

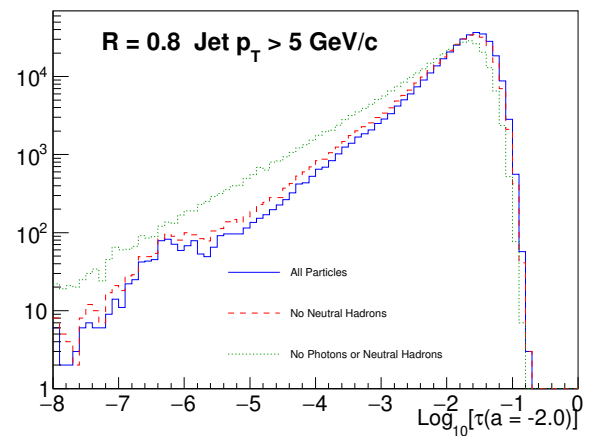

(b)

Figure 2: (a) The logarithm of angularity for jets with $R=0.8, p_{\mathrm{T}}>5 \mathrm{GeV} / c$, and $a=-2.0$ clustered from particles with transverse momenta greater than $250 \mathrm{MeV} / c$ (blue) and $500 \mathrm{GeV} / c$ (red). (b) The logarithm of angularity with the same parameters as in (a) with particle $p_{\mathrm{T}}>250 \mathrm{MeV} / c$ (blue), the angularity calculated ignoring neutrons and $K_{\mathrm{L}}^{0} \mathrm{~s}$ (red), and the angularity calculated ignoring photons in addition (green).

jet. Figure 2(b) compares the nominal angularity spectrum for jets with $R=0.8$ and $p_{\mathrm{T}}>5 \mathrm{GeV} / \mathrm{c}$ $(a=-2.0)$ with the angularity obtained by the exclusion of neutrons and $K_{\mathrm{L}}^{0} \mathrm{~s}$. It is seen that the loss of neutral hadrons has little effect on the angularity spectrum. To provide some context for the size of the observed shift, the angularity measured using only charged hadrons is also shown.

\section{Power Corrections}

The theoretical description of jet angularity depends on factorizations that are valid up to some power corrections, which can become sizable for low jet transverse momenta [9]. Because the majority of jets at an EIC will be produced at low $p_{\mathrm{T}}(<10 \mathrm{GeV} / c)$, it will be important to study how sensitive these jets are to these power corrections. This sensitivity can be explored by utilizing another definition of angularity commonly used in $e^{+} e^{-}$collisions [10]:

$$
\tau_{a}^{e^{+} e^{-}}=\frac{1}{2 E_{J}} \sum_{i \in J}\left|\vec{p}_{\mathrm{T}}^{i J}\right| \exp \left(-\left|\eta_{i J}\right|(1-a)\right)
$$

where $E_{J}$ is the jet energy, $\left|\vec{p}_{\mathrm{T}}^{i J}\right|$ is the magnitude of the $i^{\text {th }}$ particle's momentum transverse to the jet thrust axis, and $\eta_{i J}$ is the $i^{\text {th }}$ particle's rapidity with respect to the jet thrust axis. As in Eq. 2.1, ' $a$ ' is a parameter and the sum runs over all particles in the jet. The above definition of angularity is related to the definition in 2.1 according to:

$$
\tau_{a}=\left(\frac{2 E_{J}}{p_{T}}\right)^{2-a} \tau_{a}^{e^{+} e^{-}}+\mathscr{O}\left(\tau_{a}^{2}\right)
$$

where the $\mathscr{O}\left(\tau_{a}^{2}\right)$ term is exactly the power correction of interest. Thus, the sensitivity to power corrections can be assessed by comparing angularities evaluated according to 2.1 and 4.1.

Figure 3 shows the ratio $\left(\frac{2 E_{J}}{p_{T}}\right)^{2-a} \tau_{a}^{e^{+}} e^{-} / \tau_{a}$ for jet radii with $R=0.4$ and 0.8 , jet transverse momenta greater than $5 \mathrm{GeV} / c$ and $10 \mathrm{GeV} / c$, and ' $a$ ' values of $-2.0,0.0$, and 1.0. For a given 
radius and $p_{\mathrm{T}}$ range, we see that power corrections become more prominent as ' $a$ ' decreases. In addition, the power corrections are larger for larger jet radii and smaller jet transverse momenta. This information will allow future angularity analyses to avoid regions of phase space where power corrections are not under control.
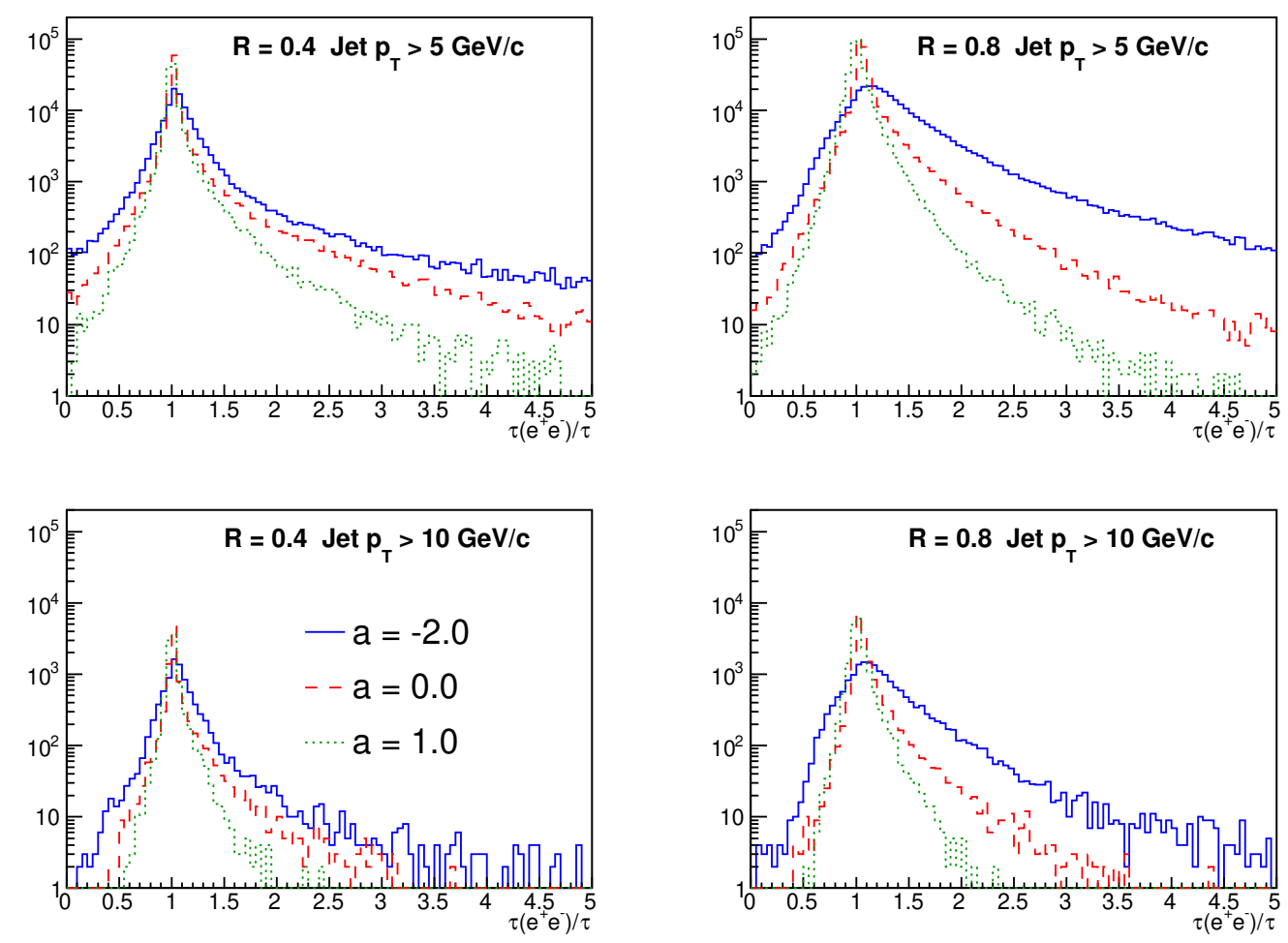

Figure 3: The ratio $\left(\frac{2 E_{J}}{p_{T}}\right)^{2-a} \tau_{a}^{e^{+} e^{-}} / \tau_{a}$ for ' $a$ ' values of -2.0 (blue), 0.0 (red), and 1.0 (green) and jets with $R=0.4$ (left column) and $R=0.8$ (right column) and transverse momenta greater than $5 \mathrm{GeV} / c$ (top row) and $10 \mathrm{GeV} / c$ (bottom row). The ratio diverges from unity as power corrections become more significant.

\section{Conclusion}

The studies presented above offer a first look at properties of the jet angularity observable in $e p$ collisions at a future EIC. Preliminary evaluations of the impact of low $p_{\mathrm{T}}$ particle acceptance and poor hadron calorimetry resolution on the angularity were carried out and the effects were seen to be modest. A scheme for the study of power corrections, the control of which will be essential for the analysis of the low $p_{\mathrm{T}}$ jets expected at an EIC, was proposed and the identification of phase space regions where power corrections are significant has been made. Comparisons of the simulated data presented here with theoretical expectations are currently underway. Future avenues of research include a more robust evaluation of detector effects using full detector simulations, and an evaluation of the sensitivity of the angularity to cold nuclear matter properties. 


\section{References}

[1] L. Asquith et al., "Jet Substructure at the Large Hadron Collider : Experimental Review," arXiv:1803.06991 [hep-ex].

[2] A. J. Larkoski, I. Moult and B. Nachman, "Jet Substructure at the Large Hadron Collider: A Review of Recent Advances in Theory and Machine Learning," arXiv:1709.04464 [hep-ph].

[3] H. A. Andrews et al., "Novel tools and observables for jet physics in heavy-ion collisions," arXiv:1808.03689 [hep-ph].

[4] T. Sjostrand, S. Mrenna and P. Z. Skands, "PYTHIA 6.4 Physics and Manual,” JHEP 0605, 026 (2006) [hep-ph/0603175].

[5] H. L. Lai et al. [CTEQ Collaboration], "Global QCD analysis of parton structure of the nucleon: CTEQ5 parton distributions,” Eur. Phys. J. C 12, 375 (2000) [hep-ph/9903282].

[6] M. Cacciari, G. P. Salam and G. Soyez, “The anti- $k_{t}$ jet clustering algorithm,” JHEP 0804, 063 (2008) [arXiv:0802.1189 [hep-ph]].

[7] M. Cacciari, G. P. Salam and G. Soyez, "FastJet User Manual,” Eur. Phys. J. C 72, 1896 (2012) [arXiv:1111.6097 [hep-ph]].

[8] L. G. Almeida, S. J. Lee, G. Perez, G. F. Sterman, I. Sung and J. Virzi, "Substructure of high-p pets at the LHC," Phys. Rev. D 79, 074017 (2009) [arXiv:0807.0234 [hep-ph]].

[9] Z. B. Kang, K. Lee and F. Ringer, "Jet angularity measurements for single inclusive jet production," JHEP 1804, 110 (2018) [arXiv:1801.00790 [hep-ph]].

[10] C. F. Berger, T. Kucs and G. F. Sterman, "Event shape / energy flow correlations," Phys. Rev. D 68, 014012 (2003) [hep-ph/0303051]. 